\title{
Editorial: Developments in Security and Privacy-Preserving Mechanisms for Future Mobile Communication Networks
}

\author{
Georgios Kambourakis • Gregorio Martinez • \\ Felix Gomez Marmol
}

Published online: 21 November 2013

(C) Springer Science+Business Media New York 2013

\section{Introduction}

The advances in wireless communication technologies toward $4 \mathrm{G}$ networks and the wide use of mobile devices have enabled users to communicate with each other and receive a wide range of mobile wireless services through various types of access networks and systems everywhere and anytime. However, the always-connected mobile device usage model brings to the foreground new security and privacy issues for both the end-user and network/service provider. In this respect, secure, reliable, and privacy-preserving communications combined with constant network availability are essential factors for the adoption of any service or application that utilize wireless technologies. Also, the tight-coupling of these wireless network domains to the Internet makes them more susceptible to attacks. New security challenges emerge constantly due to the open nature of the wireless medium, the dynamic network topology, the lack of common security/privacy policy used by network operators, the resource constraints of the mobile devices and, in some cases, the lack of a pre-deployed infrastructure.

\section{G. Kambourakis $(\bowtie)$}

Department of Information and Communication Systems

Engineering, University of the Aegean, Mytilíni, Greece

e-mail: gkamb@aegean.gr

\section{G. Martinez}

Department Information and Communications Engineering,

University of Murcia, Murcia, Spain

e-mail: gregorio@um.es

F. Gomez Marmol

NEC Laboratories Europe, Heidelberg, Germany

e-mail: felix.gomez-marmol@neclab.eu
The objective of this special issue is to foster state-ofthe-art contributions in the area of security, privacy, and its applications for future mobile communication networks. The special issue is composed of 5 rigorously peerreviewed papers covering topics such as modeling and analysis of QoS and security tradeoff in mobile platforms, covert communication channels on Android platforms and reputation management for social participatory systems, amongst others. It is expected that the special issue will stimulate further related research and technology improvements in this significant research field.

\section{Papers in this special issue}

The first manuscript, "A Model for the Analysis of QoS and Security Tradeoff in Mobile Platforms", co-authored by Ana Nieto and Javier Lopez, is based on the observation that mobile platforms are multimedia devices that provide different types of traffic and present different needs regarding security and privacy. Nevertheless, security and QoS requirements are often conflicting with each other. So, one of the main challenges lies in the identification of the reasons why security and QoS mechanisms are so related to each other. Compelled by this fact, authors present a Parametric Relationship Model (PRM) to identify the security and QoS dependencies, and to elaborate on the security and QoS tradeoff. More specifically, they perform an analysis that focuses on the mobile platform environment and also considers subjective parameters such as user's experience, which is crucial for increasing the usability of new solutions in the Future Internet.

In the next contribution, entitled "Quantifying and Classifying Covert Communication on Android", by Raquel Hill, 
Michael Hansen, and Veer Singh, authors are motivated by the fact that the exploitation of known covert channels allows modern Android applications to by-pass the built-in permission system and share data in a potentially untraceable manner. However, such hidden channels have sufficient bandwidth to transmit sensitive information (such as GPS locations) in real-time to collaborating applications with Internet access. Thus, authors elaborate on previous works involving an application layer covert communications detector. They measure the stability of the volume and vibration channels on the Android emulator, HTC G1, and Motorola Droid. Moreover, they quantify the effect their detector has on channel capacities for stealthy malicious applications using a theoretical model. A last contribution of the paper is the introduction of a new classification of covert communication for the Android platform.

The third paper, by Haleh Amintoosi and Salil S. Kanhere, entitled "A Reputation Framework for Social Participatory Sensing Systems", highlights the fact that the success of social participatory sensing is heavily based on the trustworthiness of contributions provided by participants. In this direction, authors propose an applicationagnostic reputation framework for social participatory sensing systems. The proposed framework considers both the quality of contribution and the trustworthiness level of participants within the social network. Moreover, these two aspects are further combined via a fuzzy inference system to arrive at a final trust rating for a contribution. For each participant, a reputation score is calculated as a resultant of the trust ratings assigned to them. The authors make use of the PageRank algorithm as the building block for their reputation module and present extensive simulations demonstrating the efficacy of their framework in achieving high overall trust and assigning accurate reputation scores.

The fourth contribution, "A Distributed and Collaborative Intrusion Detection Architecture for Wireless Mesh Networks", co-authored by Anderson Morais and Ana Cavalli, proposes a distributed and Collaborative Intrusion Detection System (CIDS) architecture for detecting insider attacks in WMNs at real-time, which comprises: i) a Routing Protocol Analyzer (RPA) to analyze the collected routing traffic and generate respective Routing Events; ii) a Distributed Intrusion Detection Engine (DIDE) that treats the Routing Events by applying Routing Constraints and calculates related Misbehaving Metrics; and iii) a Cooperative Consensus Mechanism (CCM) to check the Misbehaving Metrics using a proposed threshold scheme and to track down the source of intrusion. The entire CIDS solution is implemented in a virtualized mesh network platform. The experimental results suggest that the proposed CIDS architecture efficiently detects message fabrication attacks with good precision and low resource consumption.

In the fifth paper, "Quantum Secure Communication Network Protocol with Entangled Photons for Mobile Communications", by Yao-Hsin Chou, Guo-Jyun Zeng, Fang-Jhu Lin, Chi-Yuan Chen and Han-Chieh Chao, a communication protocol called Controlled Bidirectional Quantum Secret Direct Communication (CBQSDC) for mobile networks is proposed. This protocol is based on n-particle GHZ states (GreenbergerHorne-Zeilinger-states) which are transformed to EinsteinPodolsky-Rosen (EPR) pairs by entanglement swapping. GHZ states are used to carry both sides' messages and entanglement swapping could reduce the number of transmissions, thus decreasing the probability of eavesdropping. This means that in case an eavesdropper tries to steal dealer's messages, the lawful participants will perceive it and abort their transmissions.

Acknowledgments The guest editors would like to express their gratitude to the Editor in Chief Prof. Imrich Chlamtac for giving the opportunity to edit this special issue on Developments in Security and Privacypreserving mechanisms for Future Mobile Communication Networks, and Dr. Sara Fruner for making this issue published. Also, we wish to thank all the authors for submitting their work as well as the tireless reviewers who have constructively evaluated the papers within the stipulated time. Finally, we sincerely hope the reader will share our view and find this special issue very useful.

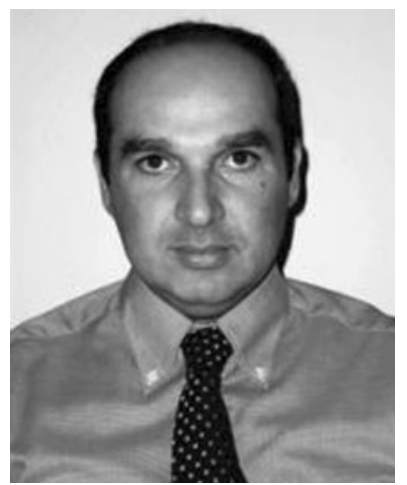

Georgios Kambourakis received the Diploma in Applied Informatics from the Athens University of Economics and Business and the $\mathrm{Ph} . \mathrm{D}$. in Information and Communication Systems Engineering from the Department of Information and Communications Systems Engineering, University of the Aegean. He also holds a Master of Education degree from the Hellenic Open University. Currently, he is an Assistant Professor at the Department of Information and Communication Systems Engineering, University of the Aegean, Greece. His research interests are in the fields of mobile and wireless networks security and privacy, VoIP security, PKI, DNS security, and mLearning and he has more than 85 publications in the above areas. He has been involved in several national and EU funded R\&D projects in the areas of Information and Communication Systems Security. $\mathrm{He}$ is also a reviewer of several IEEE and other international journals and has served as a technical program committee member for more than 100 international conferences in security and networking. 


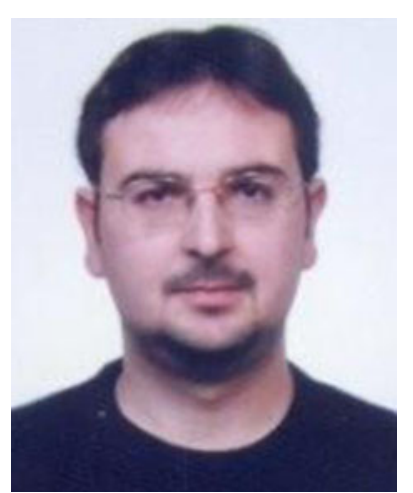

Gregorio Martinez is Associate Professor in the Department of Information and Communications Engineering of the University of Murcia. His research interests include security and management of distributed communication networks. He received the Ph.D. degree in Computer Science from the University of Murcia. He has published more than a hundred journal articles and conference papers. He has been involved as collaborator or supervisor in several opensource software projects. He is also on the editorial or review board of more than 20 international journals and guest edited more than 25 special issues. He is actively involved in research projects at both national and European level.

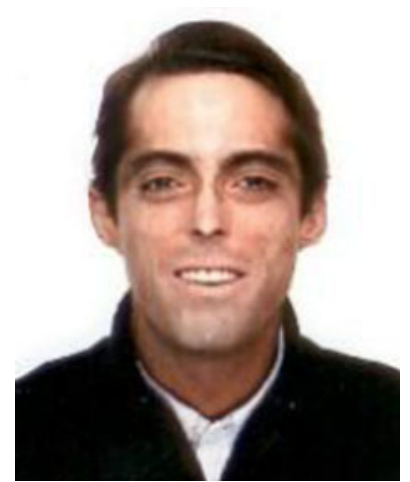

Felix Gomez Marmol is a Senior Researcher in the Security Group at NEC Laboratories Europe, Heidelberg, Germany. His research interests include authorization, authentication and trust management in distributed and heterogeneous systems, security management in mobile devices and design and implementation of security solutions for mobile and heterogeneous environments. He published over 30 research articles, holds 4 patents and participated in several special issues as guest editor in international journals. He received an $\mathrm{MSc}$ and $\mathrm{PhD}$ in computer engineering from the University of Murcia. 